\title{
An Empirical Evaluation of Salt and Pepper Noise Removal for Document Images using Median Filter
}

\author{
E. Balamurugan \\ Assistant Professor \\ Bannari Amman Institute of \\ Technology \\ Sathyamangalam \\ Tamil Nadu, India
}

\author{
P. Sengottuvelan, Ph.D \\ Associate professor \\ Bannari Amman Institute of \\ Technology \\ Sathyamangalam \\ Tamil Nadu, India
}

\author{
K. Sangeetha \\ Assistant Professor \\ Bannari Amman Institute of \\ Technology \\ Sathyamangalam \\ Tamil Nadu, India
}

\begin{abstract}
Document Image is an electronic form of paper documents it is a combination of handwritten, Machine printed texts, pictures, office documents and graphs etc. Noise reduction in document image is important to maintain the quality of images for further processing and analysis. Noise is added into an image during the time of image acquisition. Once image is captured, image pre processing is necessarily done to correct and adjust the image for further analysis tasks such as segmentation, text classification etc. Salt and Pepper noise from document images is randomly scattered white or black (or both) pixel over the image. Normally the filters are used to improve the image quality, suppress the noise. In this paper median filtering technique is proposed for removing salt and pepper noise from various types of document images and its performance is analyzed using Peak Signal to Noise Ratio (PSNR).
\end{abstract}

\section{Keywords}

Document Image, Noise reduction, Salt and Pepper Noise, Median Filtering, Peak Signal to Noise Ratio(PSNR)

\section{INTRODUCTION}

Researches on automatic processing and analysis of document images have been rapidly increased during the past few years. Document Image Analysis, including pre processing and segmentation, form processing, handwriting recognition, line drawing, map processing, and contextual processing. The document acquisition is the process of obtaining an electronic image of a paper-based document. In most cases a flat-bed scanner is used, however in digital libraries also book scanners can be considered, whereas in recent years portable devices such as digital cameras and mobile phones are used as well. Pre-processing operations in document image analysis transform the input image into an enhanced image more suitable for further analysis. Image-to image transformations in Document Analysis and Recognition (DAR) belong to four main classes: Filtering [1], Geometrical Transformations (e.g. skew detection), Object Boundary Detection and Thinning. The filtering transforms the input into an image whose value in a generic position ( $i, j$ ) is usually a function of the input values in a neighborhood of the point $(i, j)$. The three main classes of filtering operations in DAR are binarization, noise reduction, and signal enhancement [3].

Noises in an image are small, unwanted random pixels in an image where the surrounding maximum numbers of pixels are different values. This paper is discussing about one of the most popular noises in an image is the salt and pepper noise [4]. Noise in an image will decrease the performance of information of that image. Noise reduction is used to recover the ideal image from a degraded copy of an image. This paper attempts to remove Salt and Pepper noise removal in various types of document images.

The section II has an overview of Document Image. In section III the discussion is made about salt and pepper noise in section IV the discussion is made about Median Filters. In section V is about the experimental results and section VI has the conclusion of this paper.

\section{DOCUMENT IMAGES}

Document Image [9] is an electronic form of any paper document it is a combination of handwritten, Machine printed texts, pictures, Historical documents and graphs. Document images often contain mixed types of information's such as invoices, bank documents, business letters and checks. While scanning a paper document or capturing documents using cameras, the result image may contain noise from dirtiness on the page during the acquisition process. This noise may hide the translation of the image into ASCII characteristics while performing optical character recognition. It may also degrade an image such that key characteristics are damaged. So these types of images need noise reduction for further classification and segmentation

\section{SALT AND PEPPER NOISE IN IMAGES}

There are assorted types of noise [4][7] that can distort the appearance of document images. Few of them are
(a) Gaussian Noise
(b) Speckle Noise
(c) Salt \& Pepper noise
(d) Periodic Noise

Gaussian Noise is caused by random fluctuations in the signal it is modeled by random values added to an image. Speckle noise can be modeled by random values multiplied by pixel values of an image. Periodic noise is appearance when signal is subject to a periodic, rather than a random disturbance. Salt and pepper noise is also known as Impulse Noise. This noise can be caused by sharp \& sudden disturbances in the image signal. Its appearance is randomly scattered white or black (or both) pixel over the image. The main challenge in removing salt and pepper noise from binary image is due to the fact that image data as well as the noise share the same small set of values (either 0 or 1), which complicates the process of detecting and removing the noise. 


\section{MEDIAN FILTER}

Normally filters are used to remove noise from images. There are variety of filters[2] are available for salt and pepper noise removal. Filters are classified into two major types
a) Linear Filters
b) Non-linear Filters

Linear filters too tend to blur sharp edges, destroy lines and other fine image details, and perform poorly in the presence of signal-dependent noise. With non-linear filters, the noise is removed without any attempts to explicitly identify it. Median filtering is a common image enhancement technique for removing salt and pepper noise. Because this filtering is less sensitive than linear techniques to extreme changes in pixel values, it can remove salt and pepper noise without significantly reducing the sharpness of an image[6].

Median Filter $: \operatorname{Med}\{\mathrm{I}, \mathrm{Z}\}(\mathrm{P})=\operatorname{median}\{\mathrm{I}(\mathrm{q})\}$

$$
\mathrm{q} \varepsilon \operatorname{supp}(Z+\mathrm{p})
$$

This can be computed as

1. Let I be a monochrome (1-band) image.

2. Let $\mathrm{Z}$ define a neighborhood of arbitrary shape.

3. At each pixel location, $\mathrm{p}=(\mathrm{r}, \mathrm{c})$, in $\mathrm{I} \ldots$

$\rightarrow \quad$ select the $\mathrm{n}$ pixels in the Z-neighborhood of $\mathrm{p}$,

$\rightarrow \quad$ sort the $\mathrm{n}$ pixels in the neighborhood of $\mathrm{p}$, by value, into a list $L(j)$ for $j=1, \ldots, n$.

4. The output value at $\mathrm{p}$ is $\mathrm{L}(\mathrm{m})$, where $\mathrm{M}=[\mathrm{n} / 2]+1$.

The median filter considers each pixel in the image in turn and looks at its nearby neighbors to decide whether or not it is representative of its surroundings. Instead of simply replacing the pixel value with the mean of neighboring pixel values, it replaces it with the median of those values. The median is calculated by first sorting all the pixel values from the surrounding neighbourhood into numerical order and then replacing the pixel being considered with the middle pixel value. Median filtering can preserve discontinuities in a step function and can smooth a few pixels whose values differ significantly from their surroundings without affecting the other pixels.

\section{EXPERIMENTAL RESULTS}

The peak signal-to-noise ratio (PSNR) is the ratio between a signal's maximum power and the power of the signal's noise [8]. Researchers commonly use the PSNR to measure the quality of reconstructed images that have been enhanced or compressed. Each picture element (pixel) has a color value that can change when an image is compressed and then uncompressed. Signals can have a wide dynamic range, so PSNR is usually expressed in decibels, which is a logarithmic scale.

To assess the performance of the proposed Median Filter for removal of Salt $\&$ pepper noise standard performance indices are defined as follows:

Peak Signal to Noise Ratio (PSNR): It is measured in decibel $(\mathrm{dB})$ and for Gray scale image it is defined as:

$$
\mathrm{PSNR}=10 \log 10\left(255^{2} / \mathrm{MSE}\right) \mathrm{dB}
$$

In the equation "(1)", Where Mean Square Error (MSE) is the mean square error between the original and the denoised image. $\mathrm{MSE}=1 / \mathrm{mn}\left[\Sigma \Sigma(\mathrm{I}(\mathrm{i}, \mathrm{j})-\mathrm{K}(\mathrm{i}, \mathrm{j}))^{\wedge} 2\right]$

Where $\mathrm{I}$ and $\mathrm{K}$ are matrices that represent the images being compared. The two summations are performed for the dimensions " $i$ " and "j." Therefore $I(i, j)$ represents the value of pixel $(i, j)$ of image I.

The higher the PSNR in the restored image, the better is its quality. In this paper five images are experimented. The quantitative results have been given in Table-I for the different types of document images.

Table 1 MSE, PSNR Value for different types of document images

\begin{tabular}{|c|l|l|l|}
\hline S.No. & Image & MSE & PSNR \\
\hline 1. & Bank Cheque & 1.94 & 44.13 \\
\hline 2. & Hand Written & 1.81 & 42.37 \\
\hline 3. & Machine Printed & 1.96 & 45.23 \\
\hline 4. & Historical & 1.33 & 36.50 \\
\hline 5. & Office document & 1.54 & 39.61 \\
\hline
\end{tabular}




\begin{tabular}{|c|c|c|c|}
\hline Original Image & $\begin{array}{c}\text { Salt and pepper noise } \\
\text { density }(0.02)\end{array}$ & $\begin{array}{c}\text { Salt and pepper noise } \\
\text { density }(0.05)\end{array}$ & Median Filtered \\
\hline $\begin{array}{l}\text { PAYALEAT PAA ATALERAMGHES OF HOFC } \\
\text { Sample Payee Company }\end{array}$ & 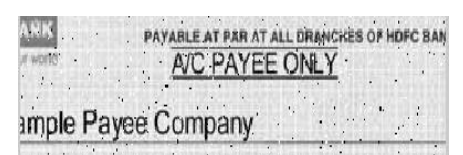 & 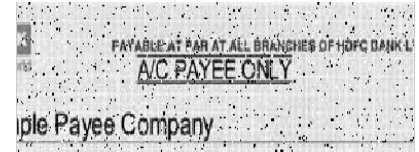 & 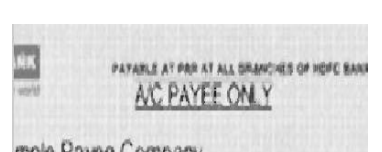 \\
\hline Es Forty Six Thousand Four Hundred Fifty & Forty Six Thousand Four Hundred Fiffy. & orty Six Thousand Four Hundred Fifty & $\begin{array}{l}\text { mole Paye Compary } \\
\text { Forty Six Thousund Four Hundred Fity }\end{array}$ \\
\hline Ind Fifty Cents Only & IFiffy Cents Drly & ifyc & Fiflycents ony \\
\hline$G A R A M O N T$ & $M O N T$ & $1 O N T$ & $G A R A M O N T$ \\
\hline 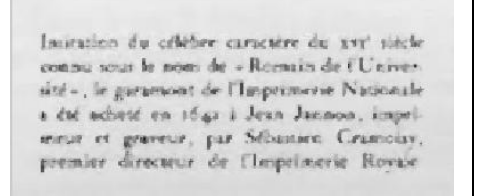 & 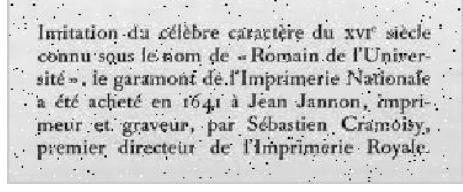 & 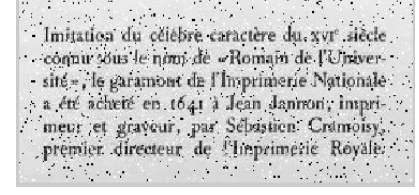 & 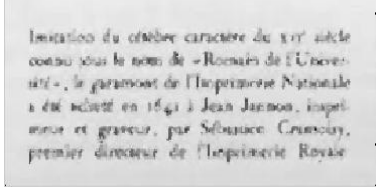 \\
\hline 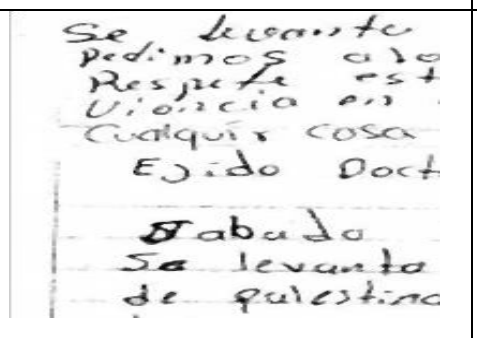 & 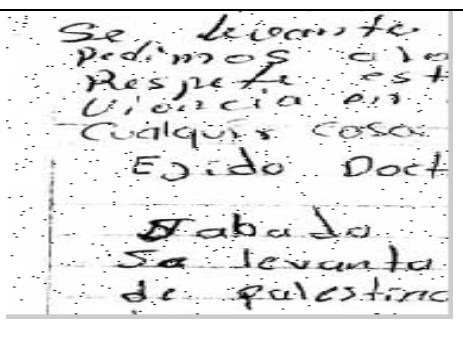 & 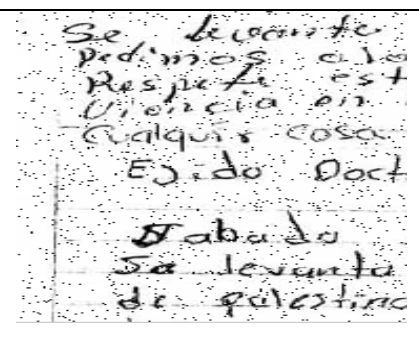 & 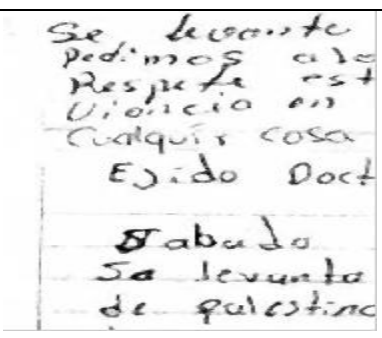 \\
\hline 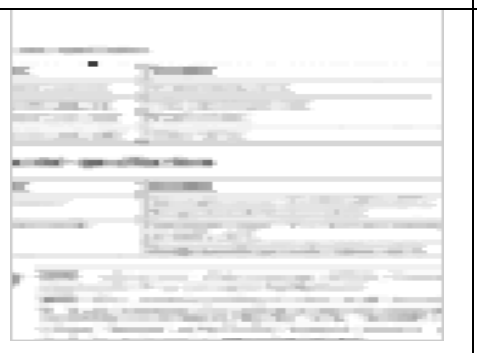 & 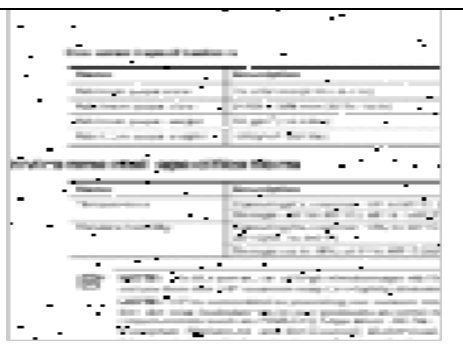 & 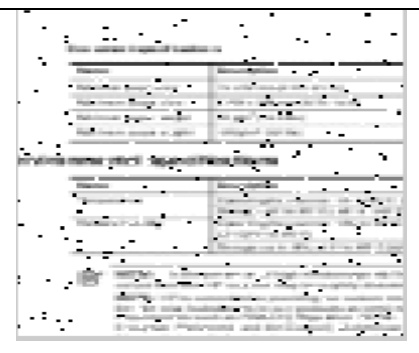 & 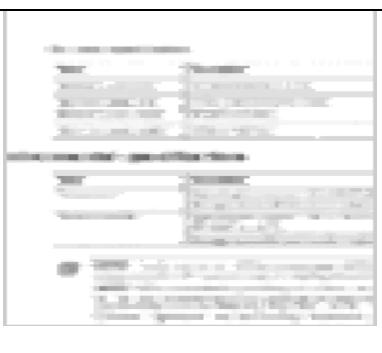 \\
\hline 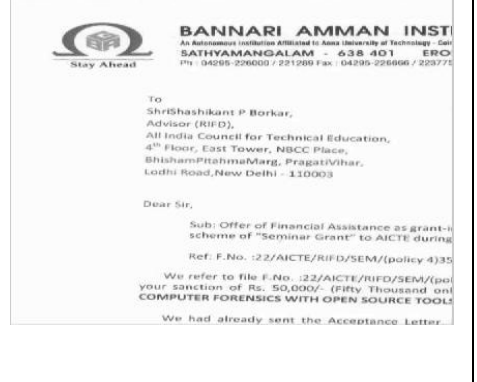 & $\begin{array}{ll}\ddots \\
\ddots\end{array}$ & 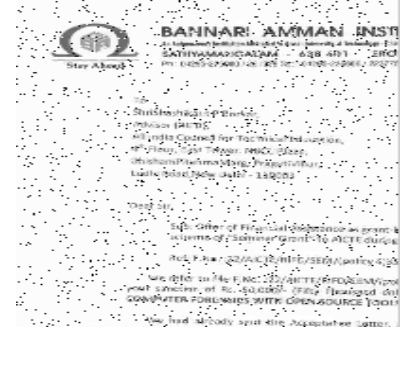 & 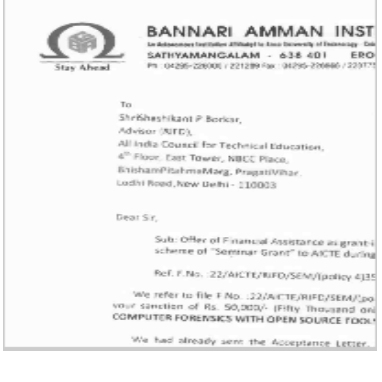 \\
\hline
\end{tabular}

Fig. 1.Comparison of different types of document images with noise density $=0.02$, noise density $=0.05$ and final result with median filtered (a) Bank Cheque image (b) Historical document (c) Handwritten document (d) Machine printed document (e) Office document 


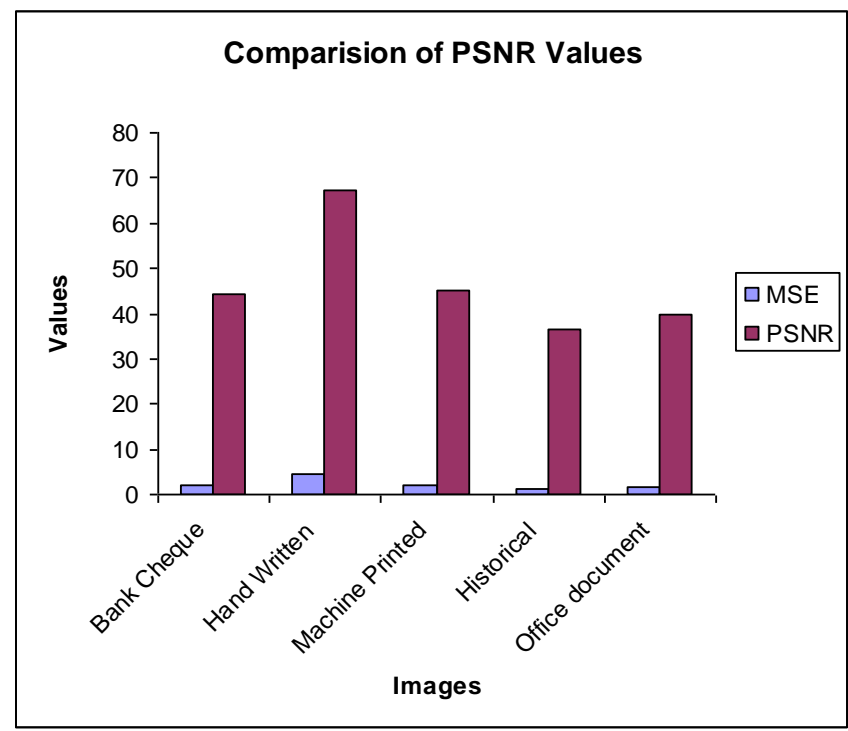

Fig. 2. Comparative graph of different images.

\section{CONCLUSION}

This paper attempts to remove Salt and pepper noise from different types of document images using median filter. The performance of the median filter with these images are compared and analyzed according to its PSNR value. From the performance analyses median filter gives better performance and results over handwritten document with clear background when compared to other bright and dark background document images.

\section{REFERENCE}

[1] Thomas s Hung et al., "A Fast Two-Dimensional Median Filtering Algorithm", IEEE Transactions on acoustics, speech, and signal processing, vol. asp-27, no. 1, February 1979.
[2] Zhou Wang and David Zhang, "A Progressive Switching Median Filter for the Removal of Impulse Noise from Highly Corrupted Images", IEEE Transactions On Circuits And Systems Ii: Analog And Digital Signal Processing, VOL. 46, NO. 1, January 1999.

[3] A. C. Bovik, T.S. Huang, and D.C. Munson, Jr., "A Generalization of Median Filtering Using Linear Combination of Order Statistics", IEEE Trans. Acoust., Speech, Signal Processing.,vol.31,pp. 1342- 1350, 1983

[4] Raymond H. Chan et al., "Salt-and-Pepper Noise Removal by Median-Type Noise Detectors and DetailPreserving Regularization", IEEE Transactions on Image Processing, VOL. 14, NO. 10, October 2005.

[5] Pei-Eng Ng; Kai-Kuang Ma, "A switching median filter with boundary discriminative noise detection for extremely corrupted images," Image Processing, IEEE Transactions on , vol.15, no.6, pp.1506,1516, June 2006 doi: 10.1109 / TIP. 2005. 871129.

[6] Shanmugavadivu P.; Jeevaraj, P.S.E.," Laplace equation based Adaptive Median Filter for highly corrupted images," Computer Communication and Informatics (ICCCI), 2012 International Conference on vol., no., pp.1,5, 10-12 Jan. 2012.

[7] Rafael C. Gonzalez and Richard E. Woods, " Digital Image Processing", 2001.

[8] Maheswari et al., "Noise Removal In Compound Image Using Median Filter," International Journal on Computer Science and Engineering Vol. 02, No. 04,2010, 1359 1362.

[9] Lawrence O Gorman, Rangachar Kasturi, "Document Image Analysis", IEEE Computer Society, 2012. 